\title{
A planar four-loop form factor in QCD
}

\author{
Vladimir A. Smirnov ${ }^{* i}$ \\ Skobeltsyn Institute of Nuclear Physics of Moscow State University, \\ 119991, Moscow, Russia \\ E-mail: smirnovetheory.sinp.msu.ru
}

\section{Johannes M. Henn}

PRISMA Cluster of Excellence, Johannes Gutenberg University, 55099 Mainz, Germany

E-mail: henn@uni-mainz.de

\section{Alexander V. Smirnov}

Research Computing Center, Moscow State University, 119991, Moscow, Russia

E-mail: asmirnov80@gmail.com

\section{Matthias Steinhauser}

Institut für Theoretische Teilchenphysik, Karlsruhe Institute of Technology(KIT),

76128 Karlsruhe, Germany

E-mail: matthias.steinhauser@kit.edu

\begin{abstract}
We compute the fermionic contribution to the photon-quark form factor to four-loop order in QCD in the planar limit in analytic form. As a by-product, the cusp and collinear anomalous dimensions are obtained. The corresponding Feynman integrals are four-loop vertex integrals with two legs on the light cone. To evaluate them we introduce an additional scale, i.e. consider only one leg on the light cone, and apply the method of differential equations.
\end{abstract}

Loops and Legs in Quantum Field Theory

24-29 April 2016

Leipzig, Germany

\footnotetext{
* Speaker.

$\dagger$ I am grateful to Matthias Staudacher for kind hospitality at the Humboldt University of Berlin during my visit before this workshop and to the organizers of the workshop for having paid my conference fee.
} 


\section{Introduction}

One of the important tasks of modern high-energy particle physics is the development of new methods to compute quantum corrections to physical cross sections. This is particularly important in the context of Quantum Chromodynamics (QCD) where higher order corrections often have a significant numerical impact. Here we discuss the evaluation [1] of a next-to-next-to-next-tonext-to-leading order $\left(\mathrm{N}^{4} \mathrm{LO}\right)$ contribution to a three-point function within QCD. We consider the photon-quark form factor, which is a building block for $\mathrm{N}^{4} \mathrm{LO}$ cross sections. Namely, it is a gauge-invariant part of virtual forth-order corrections for the process $e^{+} e^{-} \rightarrow 2$ jets, or for DrellYan production at hadron colliders.

Let $\Gamma_{q}^{\mu}$ be the photon-quark vertex function. Then the scalar form factor is defined by

$$
F_{q}\left(q^{2}\right)=-\frac{1}{4(1-\varepsilon) q^{2}} \operatorname{Tr}\left(p \nmid \Gamma_{q}^{\mu} p h \gamma_{\mu}\right)
$$

where $D=4-2 \varepsilon$ is the space-time dimension, $q=p_{1}+p_{2}$ and $p_{1}\left(p_{2}\right)$ is the incoming (anti-)quark momentum. We consider the leading order of the large- $N_{c}$ expansion of $F_{q}\left(q^{2}\right)$. As a result we only have to consider the contributions of planar Feynman diagrams.

Results for $F_{q}$ can be used to probe the infrared structure of gauge theories. Form factors encapsulate universal infrared contributions coming from soft exchanges between two partons. The general form of the latter is known [2, 3, 4, 5, 6, 7] and depends on cusp and collinear anomalous dimensions.

Two-loop corrections to $F_{q}$ have been computed more than 25 years ago $[8,9,10,11]$. The first three-loop result has been presented in Ref. [12] and has later been confirmed in Ref. [13]. Analytic results for the three-loop form factor integrals were presented in Ref. [14]. In Ref. [15], the results of Ref. [14] have been used to compute $F_{q}$ at three loops up to order $\varepsilon^{2}$, i.e., transcendental weight eight, as a preparation for the four-loop calculation.

In our calculation we obtain the fermionic corrections to $F_{q}$ in the large- $N_{c}$ limit, to the fourloop order. Let us emphasize that the time passed between the evaluation of three-loop corrections and the first four-loop correction is essentially less than the corresponding difference between twoand three-loop calculations. It looks like this happened because of the development of powerful methods to evaluate multiloop Feynman integrals.

Other attempts to calculate similar form factors or master integrals were reported on in Refs. [16, 17, 18]. The evaluation of the master integrals in Refs. [16, 17] was performed only by numerical methods while Ref. [18] presents results only for some individual integrals in an analytical form.

In the next section we briefly outline our calculation and present results for the form factor and for the cusp and collinear anomalous dimensions. The next section is dedicated to the classification and evaluation of the master integrals. Then we present our conclusions.

\section{Results}

We generate the Feynman amplitudes with the help of qgraf [19] and transform the output to FORM $[20,21]$ notation using $q 2 e$ and $\exp [22,23]$. For the reduction to master integrals we use the program FIRE $[24,25,26]$ which we apply in combination with LiteRed [27, 28]. Relations 
between primary master integrals occurring in the reduction tables are revealed with the help of tsort, which is part of the latest FIRE version [26], and based on ideas presented in Ref. [25]. This leads to 78 master integrals needed for the fermionic part. More generally, we find that a total of 99 master integrals are sufficient for arbitrary planar integrals. They are all computed as described in the next section.

In our calculation we allow for a generic QCD gauge parameter $\xi$ and expand the Feynman diagrams around $\xi=0$, which corresponds to Feynman gauge, up to linear order. We checked that $\xi$ drops out before inserting explicit results for the master integrals.

In the following we present results for the form factor $F_{q}$ and the related anomalous dimensions. $F_{q}$ is conveniently shown in terms of the bare strong coupling constant. In that case the perturbative expansion of $F_{q}$ can be cast in the form

$$
F_{q}=1+\sum_{n \geq 1}\left(\frac{\alpha_{s}^{0}}{4 \pi}\right)^{n}\left(\frac{\mu^{2}}{-q^{2}}\right)^{(n \varepsilon)} F_{q}^{(n)} .
$$

Analytic results for $F_{q}^{(n)}$, with $n \leq 3$, expanded in $\varepsilon$ up to transcendental weight eight can be found in Ref. [15]. We refrain from repeating them here.

The main result of this letter is the fermionic contribution to $F_{q}^{(4)}$ in the large- $N_{c}$ limit. It is given by

$$
\begin{aligned}
& \left.F_{q}^{(4)}\right|_{\text {large- } N_{c}}= \\
& \frac{1}{\varepsilon^{7}}\left[\frac{1}{12} N_{c}^{3} n_{f}\right]+\frac{1}{\varepsilon^{6}}\left[\frac{41}{648} N_{c}^{2} n_{f}^{2}-\frac{37}{648} N_{c}^{3} n_{f}\right]+\frac{1}{\varepsilon^{5}}\left[\frac{1}{54} N_{c} n_{f}^{3}+\frac{277}{972} N_{c}^{2} n_{f}^{2}\right. \\
& \left.+\left(\frac{41 \pi^{2}}{648}-\frac{6431}{3888}\right) N_{c}^{3} n_{f}\right]+\frac{1}{\varepsilon^{4}}\left[\left(\frac{215 \zeta_{3}}{108}-\frac{72953}{7776}-\frac{227 \pi^{2}}{972}\right) N_{c}^{3} n_{f}\right. \\
& \left.+\frac{11}{54} N_{c} n_{f}^{3}+\left(\frac{5}{24}+\frac{127 \pi^{2}}{1944}\right) N_{c}^{2} n_{f}^{2}\right]+\frac{1}{\varepsilon^{3}}\left[\left(\frac{229 \zeta_{3}}{486}-\frac{630593}{69984}+\frac{293 \pi^{2}}{2916}\right) N_{c}^{2} n_{f}^{2}\right. \\
& \left.+\left(\frac{2411 \zeta_{3}}{243}-\frac{1074359}{69984}-\frac{2125 \pi^{2}}{1296}+\frac{413 \pi^{4}}{3888}\right) N_{c}^{3} n_{f}+\left(\frac{127}{81}+\frac{5 \pi^{2}}{162}\right) N_{c} n_{f}^{3}\right] \\
& +\frac{1}{\varepsilon^{2}}\left[\left(-\frac{41 \zeta_{3}}{81}+\frac{29023}{2916}+\frac{55 \pi^{2}}{162}\right) N_{c} n_{f}^{3}+\left(\frac{11684 \zeta_{3}}{729}-\frac{41264407}{419904}-\frac{155 \pi^{2}}{72}\right.\right. \\
& \left.+\frac{2623 \pi^{4}}{29160}\right) N_{c}^{2} n_{f}^{2}+\left(-\frac{537625 \zeta_{3}}{11664}-\frac{599 \pi^{2} \zeta_{3}}{486}+\frac{12853 \zeta_{5}}{180}+\frac{155932291}{839808}\right. \\
& \left.\left.-\frac{27377 \pi^{2}}{69984}-\frac{1309 \pi^{4}}{7290}\right) N_{c}^{3} n_{f}\right]+\frac{1}{\varepsilon}\left[\left(-\frac{451 \zeta_{3}}{81}+\frac{331889}{5832}+\frac{635 \pi^{2}}{243}+\frac{151 \pi^{4}}{4860}\right) N_{c} n_{f}^{3}\right. \\
& +\left(\frac{661 \zeta_{3}}{4}-\frac{1805 \pi^{2} \zeta_{3}}{729}+\frac{19877 \zeta_{5}}{405}-\frac{608092805}{839808}-\frac{6041473 \pi^{2}}{209952}+\frac{8263 \pi^{4}}{21870}\right) N_{c}^{2} n_{f}^{2} \\
& +\left(-\frac{5427821 \zeta_{3}}{5832}+\frac{48563 \pi^{2} \zeta_{3}}{2916}-\frac{1373 \zeta_{3}^{2}}{324}+\frac{12847 \zeta_{5}}{810}+\frac{662170621}{279936}+\frac{17271517 \pi^{2}}{209952}\right.
\end{aligned}
$$




$$
\begin{aligned}
& \left.\left.-\frac{78419 \pi^{4}}{25920}+\frac{21625 \pi^{6}}{81648}\right) N_{c}^{3} n_{f}\right]+\left[\left(-\frac{10414 \zeta_{3}}{243}-\frac{205 \pi^{2} \zeta_{3}}{243}-\frac{1097 \zeta_{5}}{135}+\frac{10739263}{34992}\right.\right. \\
& \left.+\frac{145115 \pi^{2}}{8748}+\frac{1661 \pi^{4}}{4860}\right) N_{c} n_{f}^{3}+\left(\frac{65735207 \zeta_{3}}{52488}-\frac{4262 \pi^{2} \zeta_{3}}{2187}-\frac{71711 \zeta_{3}^{2}}{1458}\right. \\
& \left.+\frac{725828 \zeta_{5}}{1215}-\frac{68487272627}{15116544}-\frac{295056623 \pi^{2}}{1259712}-\frac{889 \pi^{4}}{6480}+\frac{43559 \pi^{6}}{204120}\right) N_{c}^{2} n_{f}^{2} \\
& +\left(-\frac{1774255975 \zeta_{3}}{209952}+\frac{265217 \pi^{2} \zeta_{3}}{3888}-\frac{2692 \pi^{4} \zeta_{3}}{3645}+\frac{973135 \zeta_{3}^{2}}{1458}-\frac{56656921 \zeta_{5}}{19440}\right. \\
& -\frac{58657 \pi^{2} \zeta_{5}}{1620}+\frac{1643545 \zeta_{7}}{1008}+\frac{555003607961}{30233088}+\frac{785989381 \pi^{2}}{839808}-\frac{34077673 \pi^{4}}{2099520} \\
& \left.\left.-\frac{146197 \pi^{6}}{612360}\right) N_{c}^{3} n_{f}\right]+\ldots,
\end{aligned}
$$

where the ellipses stand for $n_{f}$-independent contributions.

The cusp and collinear anomalous dimension is conveniently extracted from $\log \left(F_{q}\right)$ (after renormalization of $\alpha_{s}$ ). The coefficients of the cusp and collinear anomalous dimensions are defined through

$$
\gamma_{x}=\sum_{n \geq 0}\left(\frac{\alpha_{s}\left(\mu^{2}\right)}{4 \pi}\right)^{n} \gamma_{x}^{n}
$$

with $x \in\{$ cusp, q $\}$.

The anomalous dimension $\gamma_{\text {cusp }}$ can be extracted from the coefficient of the quadratic, and $\gamma_{q}$ from the first-order pole in $\varepsilon$. In the large- $N_{c}$ limit we obtain for $\gamma^{\text {cusp }}$

$$
\begin{aligned}
\gamma_{\text {cusp }}^{0}= & 4 \\
\gamma_{\text {cusp }}^{1}= & \left(-\frac{4 \pi^{2}}{3}+\frac{268}{9}\right) N_{c}-\frac{40 n_{f}}{9}, \\
\gamma_{\text {cusp }}^{2}= & \left(\frac{44 \pi^{4}}{45}+\frac{88 \zeta_{3}}{3}-\frac{536 \pi^{2}}{27}+\frac{490}{3}\right) N_{c}^{2}+\left(-\frac{64 \zeta_{3}}{3}+\frac{80 \pi^{2}}{27}-\frac{1331}{27}\right) N_{c} n_{f} \\
& -\frac{16 n_{f}^{2}}{27}, \\
\gamma_{\text {cusp }}^{3}= & \left(-\frac{32 \pi^{4}}{135}+\frac{1280 \zeta_{3}}{27}-\frac{304 \pi^{2}}{243}+\frac{2119}{81}\right) N_{c} n_{f}^{2}+\left(\frac{128 \pi^{2} \zeta_{3}}{9}+224 \zeta_{5}-\frac{44 \pi^{4}}{27}\right. \\
& \left.-\frac{16252 \zeta_{3}}{27}+\frac{13346 \pi^{2}}{243}-\frac{39883}{81}\right) N_{c}^{2} n_{f}+\left(\frac{64 \zeta_{3}}{27}-\frac{32}{81}\right) n_{f}^{3}+\ldots .
\end{aligned}
$$

where the ellipses in $\gamma_{\text {cusp }}^{3}$ indicate non- $n_{f}$ terms which are not yet known. For $\gamma^{q}$ we have

$$
\begin{aligned}
& \gamma_{q}^{0}=-\frac{3 N_{c}}{2} \\
& \gamma_{q}^{1}=\left(\frac{\pi^{2}}{6}+\frac{65}{54}\right) N_{c} n_{f}+\left(7 \zeta_{3}-\frac{5 \pi^{2}}{12}-\frac{2003}{216}\right) N_{c}^{2} \\
& \gamma_{q}^{2}=\left(-\frac{\pi^{4}}{135}-\frac{290 \zeta_{3}}{27}+\frac{2243 \pi^{2}}{972}+\frac{45095}{5832}\right) N_{c}^{2} n_{f}+\left(-\frac{4 \zeta_{3}}{27}-\frac{5 \pi^{2}}{27}+\frac{2417}{1458}\right) N_{c} n_{f}^{2}
\end{aligned}
$$




$$
\begin{aligned}
+ & N_{c}^{3}\left(-68 \zeta_{5}-\frac{22 \pi^{2} \zeta_{3}}{9}-\frac{11 \pi^{4}}{54}+\frac{2107 \zeta_{3}}{18}-\frac{3985 \pi^{2}}{1944}-\frac{204955}{5832}\right), \\
\gamma_{q}^{3}= & N_{c}^{3}\left[\left(-\frac{680 \zeta_{3}^{2}}{9}-\frac{1567 \pi^{6}}{20412}+\frac{83 \pi^{2} \zeta_{3}}{9}+\frac{557 \zeta_{5}}{9}+\frac{3557 \pi^{4}}{19440}-\frac{94807 \zeta_{3}}{972}+\frac{354343 \pi^{2}}{17496}\right.\right. \\
& \left.\left.+\frac{145651}{1728}\right) n_{f}\right]+\left(-\frac{8 \pi^{4}}{1215}-\frac{356 \zeta_{3}}{243}-\frac{2 \pi^{2}}{81}+\frac{18691}{13122}\right) N_{c} n_{f}^{3}+\left(-\frac{2}{3} \pi^{2} \zeta_{3}\right. \\
& \left.+\frac{166 \zeta_{5}}{9}+\frac{331 \pi^{4}}{2430}-\frac{2131 \zeta_{3}}{243}-\frac{68201 \pi^{2}}{17496}-\frac{82181}{69984}\right) N_{c}^{2} n_{f}^{2}+\ldots
\end{aligned}
$$

The expressions in Eqs. (2.4) and (2.5) up to three-loop order confirm the results in the literature $[30,31,32,33,12,29,13]$ and the $N_{c}^{3} n_{f}^{3}$ term of $\gamma_{\text {cusp }}^{3}$ agrees with the result of Ref. [34, 35]. All other terms in the four-loop results $\gamma_{\text {cusp }}^{3}$ and $\gamma_{q}^{3}$ are new. The constant $n_{f}^{2}$ term of $\gamma_{\text {cusp }}^{3}$ in Eq. (2.4) is also in agreement with a recent calculation of Ref. [36].

\section{Evaluating master integrals}

In our calculation, we are dealing with the following family of planar Feynman integrals:

$$
\begin{aligned}
F & a_{1}, \ldots, a_{18}=\int \ldots \int \frac{\mathrm{d}^{D} k_{1} \ldots \mathrm{d}^{D} k_{4}}{\left(-\left(k_{1}+p_{1}\right)^{2}\right)^{a_{1}}\left(-\left(k_{2}+p_{1}\right)^{2}\right)^{a_{2}}\left(-\left(k_{3}+p_{1}\right)^{2}\right)^{a_{3}}\left(-\left(k_{4}+p_{1}\right)^{2}\right)^{a_{4}}} \\
& \times \frac{1}{\left(-\left(k_{1}-p_{2}\right)^{2}\right)^{a_{5}}\left(-\left(k_{2}-p_{2}\right)^{2}\right)^{a_{6}}\left(-\left(k_{3}-p_{2}\right)^{2}\right)^{a_{7}}\left(-\left(k_{4}-p_{2}\right)^{2}\right)^{a_{8}}\left(-k_{1}^{2}\right)^{a_{9}}\left(-k_{2}^{2}\right)^{a_{10}}} \\
& \times \frac{1}{\left(-k_{3}^{2}\right)^{a_{11}}\left(-k_{4}^{2}\right)^{a_{12}}\left(-\left(k_{1}-k_{2}\right)^{2}\right)^{a_{13}}\left(-\left(k_{1}-k_{3}\right)^{2}\right)^{a_{14}}\left(-\left(k_{1}-k_{4}\right)^{2}\right)^{a_{15}}} \\
& \times \frac{1}{\left(-\left(k_{2}-k_{3}\right)^{2}\right)^{a_{16}}\left(-\left(k_{2}-k_{4}\right)^{2}\right)^{a_{17}}\left(-\left(k_{3}-k_{4}\right)^{2}\right)^{a_{18}}} .
\end{aligned}
$$

with $p_{1}^{2}=p_{2}^{2}=0, q^{2} \equiv p_{3}^{2}=\left(p_{1}+p_{2}\right)^{2}$. This family can be decomposed into several subfamilies whete certain subsets of the 12 indices can be positive.

After solving integration-by-parts identities [37] with the latest version of the program F IRE [24, 25, 26] we reveal 99 master integrals. To evaluate them analytically we follow the idea of Ref. [38] and introduce an additional scale considering one more leg off the light cone, i.e. $p_{2}^{2} \neq 0$. This enables us to apply the powerful machinery of the method of differential equations [39, 40, 41, 42] by deriving and then solving differential equations with respect to $x=p_{2}^{2} / p_{3}^{2}$.

For this family of integrals which are functions of $x$, FIRE gives 504 master integrals. Then we follow the strategy of Ref. [42] (see also [43]) where it was suggested to turn to a so-called canonical basis. We used the recipes formulated in $[42,43]$ to achieve this goal. In particular, it $\mathrm{s}$ helpful to select basis integrals that have constant leading singularities [44].

The system of differential equations in our canonical basis $f$ has the following form

$$
\partial_{x} f(x, \varepsilon)=\varepsilon\left[\frac{a}{x}+\frac{b}{1-x}\right] f(x, \varepsilon),
$$

where $a$ and $b$ are some constant (i.e. $x$ - and $\varepsilon$-independent) $504 \times 504$ matrices. The special features of this form are the manifest Fuchsian property of the singularities, i.e. only single poles 
in $x=0,1, \infty$ are present on the right-hand side of Eq. (3.2), and the fact that the right-hand side is proportional to $\varepsilon$. The latter property can be achieved for iterated integrals. Here, it implies that the solution, to any order in $\varepsilon$, can be written in terms of iterated integrals over the kernels $\mathrm{d} x / x$ and $\mathrm{d} x /(x-1)$, i.e. in terms of harmonic polylogarithms [45].

We fix the boundary values at $x=1$ by demanding regularity of the integrals in this limit and using explicit results for some propagator type integrals. They can be determined easily: in most cases, the boundary value is zero due to kinematical factors. Otherwise one can use results for propagator type integrals available in the literature, see, in particular, four-loop analytic results in $[46,47,48]$.

We then use the differential equation (3.2) to transport this boundary value back to $x=0$. (In mathematical language, we construct the Drinfeld associator, perturbatively in $\varepsilon$.) Finally, unlike the $x \rightarrow 1$ limit, the $x \rightarrow 0$ limit is singular, in the sense that the matrix exponential $x^{\varepsilon a}$ contains several terms $x^{\varepsilon \alpha}$, with $\alpha \neq 0$. These non-zero values of $\alpha$ correspond to contributions of various regions $[49,50,51]$ to the asymptotic expansion in the given limit. The on-shell integrals we would like to compute correspond to the so-called "hard" region with $\alpha=0$.

In order to determine to the on-shell integrals, we reduce the basis $f$ for on-shell kinematics, expressing it in terms of 99 on-shell master integrals. We then match the expression so obtained to the hard region at $x=0$. We find that this determines all the 99 integrals (naturally, some of the 504 equations are redundant). In order to carry out these algebraic manipulations, we successfully applied the Mathematica package HPL.m [52].

Our results for all the master integrals, both with two legs on the light cone and with one leg on the light cone will be published elsewhere. This is an example of our result for the integral $F_{0,0,1,1,1,1,0,1,0,1,1,-2,1,1,1,1,0,1}$, in the notation of Eq. 3.1,

$$
\begin{aligned}
& \frac{1}{576 \varepsilon^{8}}+\frac{1}{216} \pi^{2} \frac{1}{\varepsilon^{6}}+\frac{151}{864} \zeta_{3} \frac{1}{\varepsilon^{5}}+\frac{173}{10368} \pi^{4} \frac{1}{\varepsilon^{4}}+\left[\frac{505}{1296} \pi^{2} \zeta_{3}+\frac{5503}{1440} \zeta_{5}\right] \frac{1}{\varepsilon^{3}} \\
& +\left[\frac{6317}{155520} \pi^{6}+\frac{9895}{2592} \zeta_{3}^{2}\right] \frac{1}{\varepsilon^{2}}+\left[\frac{89593}{77760} \pi^{4} \zeta_{3}+\frac{3419}{270} \pi^{2} \zeta_{5}-\frac{169789}{4032} \zeta_{7}\right] \frac{1}{\varepsilon} \\
& +\left[\frac{407}{15} s_{8 a}+\frac{41820167}{653184000} \pi^{8}+\frac{41719}{972} \pi^{2} \zeta_{3}^{2}-\frac{263897}{2160} \zeta_{3} \zeta_{5}\right]+\ldots,
\end{aligned}
$$

where $s_{8 a}=\sum_{i_{1}=1}^{\infty} \frac{1}{i_{1}^{5}} \sum_{i_{2}=1}^{i_{1}} \frac{1}{i_{2}^{3}}=\zeta_{8}+\zeta_{5,3}=1.041785 \ldots$ and $\zeta_{5,3}$ is a multiple zeta value [52]

\section{Conclusion}

A natural extension of this work is to apply the planar master integrals we computed to evaluate the non-fermionic planar contribution, where the integral reduction is more complicated. The master integrals involved in this calculation are the 99 master integrals which we have already evaluated. The integration-by-parts reduction tunrs out to be more complicated. A typical time scale for a reduction job for a individual family of integrals (where indices for a specific subset consisting of 12 indices in (3.1) can be positive) in the present calculation was one month while for the non-fermionic planar contribution, more time is required. Furthermore, we expect that the methods discussed in this paper can also be applied to non-planar form factor integrals. 


\section{References}

[1] J. M. Henn, A. V. Smirnov, V. A. Smirnov and M. Steinhauser, JHEP 1605 (2016) 066.

[2] A. H. Mueller, Phys. Rev. D20 (1979) 2037.

[3] J. C. Collins, Phys. Rev. D22 (1980) 1478.

[4] A. Sen, Phys. Rev. D24 (1981) 3281.

[5] L. Magnea and G. F. Sterman, Phys. Rev. D42 (1990) 4222.

[6] I. A. Korchemskaya and G. P. Korchemsky, Phys. Lett. B287 (1992) 169.

[7] G. F. Sterman and M. E. Tejeda-Yeomans, Phys. Lett. B552 (2003) 48.

[8] G. Kramer and B. Lampe, Z. Phys. C34 (1987) 497. [Erratum: Z. Phys.C42,504(1989)].

[9] T. Matsuura and W. L. van Neerven, Z. Phys. C38 (1988) 623.

[10] T. Matsuura, S. C. van der Marck, and W. L. van Neerven, Nucl. Phys. B319 (1989) 570.

[11] T. Gehrmann, T. Huber, and D. Maitre, Phys. Lett. B622 (2005) 295.

[12] P. A. Baikov, K. G. Chetyrkin, A. V. Smirnov, V. A. Smirnov, and M. Steinhauser, Phys. Rev. Lett. 102 (2009) 212002.

[13] T. Gehrmann, E. W. N. Glover, T. Huber, N. Ikizlerli, and C. Studerus, JHEP 06 (2010) 094.

[14] R. N. Lee and V. A. Smirnov, JHEP 02 (2011) 102.

[15] T. Gehrmann, E. W. N. Glover, T. Huber, N. Ikizlerli, and C. Studerus, JHEP 11 (2010) 102.

[16] R. H. Boels, B. A. Kniehl, O. V. Tarasov, and G. Yang, JHEP 02 (2013) 063.

[17] R. Boels, B. A. Kniehl, and G. Yang, Nucl. Phys. B902 (2016) 387.

[18] A. von Manteuffel, E. Panzer and R. M. Schabinger, Phys. Rev. D 93 (2016) no.12, 125014.

[19] P. Nogueira, J. Comput. Phys. 105 (1993) 279.

[20] J. A. M. Vermaseren, math-ph/0010025.

[21] J. Kuipers, T. Ueda, J. A. M. Vermaseren, and J. Vollinga, Comput. Phys. Commun. 184 (2013) 1453.

[22] R. Harlander, T. Seidensticker, and M. Steinhauser, Phys. Lett. B426 (1998) 125.

[23] T. Seidensticker, in AIHENP 99, Heraklion, Crete, Greece, April 12-16, 1999, 1999, hep-ph/9905298.

[24] A. V. Smirnov, JHEP 10 (2008) 107.

[25] A. V. Smirnov and V. A. Smirnov, Comput. Phys. Commun. 184 (2013) 2820.

[26] A. V. Smirnov, Comput. Phys. Commun. 189 (2014) 182,

[27] R. N. Lee, arXiv:1212.2685.

[28] R. N. Lee, J. Phys. Conf. Ser. 523 (2014) 012059.

[29] T. Becher and M. Neubert, JHEP 06 (2009) 081.

[30] A. Vogt, Phys. Lett. B497 (2001) 228.

[31] C. F. Berger, Phys. Rev. D66 (2002) 116002.

[32] S. Moch, J. A. M. Vermaseren, and A. Vogt, Nucl. Phys. B688 (2004) 101. 
[33] S. Moch, J. A. M. Vermaseren, and A. Vogt, Phys. Lett. B625 (2005) 245.

[34] J. A. Gracey, Phys. Lett. B322 (1994) 141.

[35] M. Beneke and V. M. Braun, Nucl. Phys. B454 (1995) 253.

[36] B. Ruijl, T. Ueda, J. A. M. Vermaseren, J. Davies and A. Vogt, arXiv:1605.08408 [hep-ph].

[37] K. G. Chetyrkin and F. V. Tkachov, Nucl. Phys. B192 (1981) 159.

[38] J. M. Henn, A. V. Smirnov, and V. A. Smirnov, JHEP 1403 (2014) 088.

[39] A. V. Kotikov, Phys. Lett. B254 (1991) 158.

[40] E. Remiddi, A110 (1997) 1435.

[41] T. Gehrmann and E. Remiddi, Nucl. Phys. B580 (2000) 485.

[42] J. M. Henn, Phys. Rev. Lett. 110 (2013), no. 25251601.

[43] J. M. Henn, J. Phys. A48 (2015), no. 15153001.

[44] F. Cachazo, arXiv:0803.1988.

[45] E. Remiddi and J. A. M. Vermaseren, Int. J. Mod. Phys. A15 (2000) 725.

[46] P. A. Baikov and K. G. Chetyrkin, Nucl. Phys. B837 (2010) 186.

[47] R. N. Lee, A. V. Smirnov, and V. A. Smirnov, Nucl. Phys. B856 (2012) 95.

[48] E. Panzer, arXiv:1506.0724.

[49] M. Beneke and V. A. Smirnov, Nucl. Phys. B522 (1998) 321.

[50] V. A. Smirnov and E. R. Rakhmetov, Theor. Math. Phys. 120 (1999) 870.

[51] V. A. Smirnov, Applied asymptotic expansions in momenta and masses, Springer Tracts Mod. Phys. 177 (2002) 1-262.

[52] D. Maître, Comput. Phys. Commun. 174 (2006) 222. 